allowed the aqueous to penetrate and ultimately dissolve the whole lens substance? The swelling of the lens during the earlier stages might bring the lens capsule against the iris, with the result that the pattern of its uvea remained as a permanent appearance. It seems a possible explanation.

\title{
A CLINICAL NOTE ON GLAUCOMA
}

\author{
BY \\ E. E. Maddox \\ BOURNEMOUTH
}

THE following simple addition to our ordinary measures for reducing the tension in most cases of glaucoma seems worth communicating.

About five years ago I was much interested to discover that high frequency currents had the effect of almost immediately lowering the tension in nearly all moderately hypertense eyes.

In one case only during these years has an untoward sequel resulted. The patient had toxic cyclitis with secondary glaucoma, and the treatment was followed by a temporary increase of hardness and pain. It was this case that made me delay publication till further experience should prove the method safe enough to recommend. Caution, therefore, should be observed with inflamed eyes. With very hard and strangulated eyes the effect may be nil.

The application need not be longer than from one to four minutes, and although the effect is more or less transitory, I find that in most cases, repeated applications do good in the long run. This indeed is what we might expect, for a temporary reduction of tension must allow the oppressed structures a breathing space in which to recover themselves from the continuous pressure, thus allowing a flushing with fresh blood and consequent increase of nourishment.

Until recently I have applied the current, either directly or through the operator's fingers, to the eyeball itself through the closed lids ; but now find it makes little difference if the application is made instead against the temporal margin of the orbit. In order not to startle the patient, the current should not be turned on until contact has been made with the skin, as otherwise slight sparking is caused. In any case the patient may be assured that there will be no shock.

The lowering of the general blood pressure by ordinary high frequency currents applied to the body has long been a matter of common knowledge, but the local effect of these weak currents on glaucoma does not appear to have attracted attention, and will, I hope, prove a useful addition to our ophthalmic armamentarium. 